UCSD/PTH 14-06

\title{
All-fermion electrodynamics and fermion number anomaly inflow
}

\author{
S. .M. Kravec ${ }^{a}$, John McGreevy ${ }^{a}$ and Brian Swingle S,c $^{b}$ \\ a Department of Physics, University of California at San Diego, La Jolla, CA 92093, \\ $U S A$ \\ ${ }^{b}$ Department of Physics, Harvard University, Cambridge, MA 02138, USA \\ ${ }^{c}$ Department of Physics, Stanford University, Stanford, CA 94305, USA
}

\begin{abstract}
We demonstrate that 3+1-dimensional quantum electrodynamics with fermionic charges, fermionic monopoles, and fermionic dyons arises at the edge of a $4+1$ dimensional gapped state with short-range entanglement. This state cannot be adiabatically connected to a product state, even in the absence of any symmetry. This provides independent evidence for the obstruction found by [1] to a $3+1$-dimensional short-distance completion of all-fermion electrodynamics. The nontriviality of the bulk is demonstrated by a novel fermion number anomaly.
\end{abstract}

September 2014 


\section{Contents}

1 Introduction 2

2 The $B d C$ model coupled to matter 5

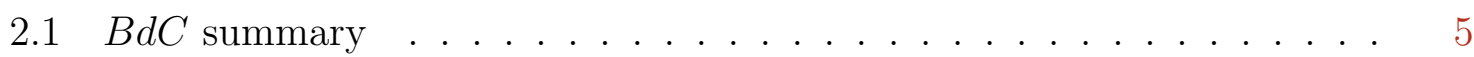

2.2 Coupling to strings (matter) . . . . . . . . . . . . . 8

2.3 Edge physics. . . . . . . . . . . . . . . . . 8

3 The Bad Thing that Happens on $\mathbb{C P}^{2} \quad 10$

4 Coupled Layer Construction $\quad 14$

4.1 Warmup: deconstruction of lattice electrodynamics . . . . . . . . . 15

4.2 Dyon string condensation in more detail . . . . . . . . . . . . 17

4.3 Alternative description of layer construction . . . . . . . . . . . . . 18

4.4 Extension to $D=3+1$ and derivation of $B F$ theory . . . . . . . . . 20

5 Fermion number anomaly inflow 20

6 Consequences for all-fermion toric code $\quad 22$

A Lattice bosons for duality-symmetric surface QED 27

B More details on monopole strings and vortex sheets in $5 \mathrm{~d}$ abelian gauge theory 


\section{Introduction}

Symmetry protected topological states (SPTs) with symmetry group $G$ are quantum phases of matter which cannot be adiabatically connected to the trivial phase in the presence of the symmetry $G$, but which can be adiabatically connected to the trivial phase if the symmetry $G$ is broken. Absent any symmetry, the lack of adiabatic connection to a product state generally implies topological order: long-ranged entanglement and topology-dependent groundstate degeneracy (for a review, see e.g. [2]). However, in rare cases, short-range entangled topological states can be non-trivial in the absence of any symmetry [3]. For example, $2+1$-dimensional chiral states are distinguished from the trivial phase by their gapless chiral edge modes which persist in the absence of any symmetry. In this paper, we give another example of a short-range entangled bulk topological state not protected by any symmetry. It is made from bosons in $4+1$ dimensions and its edge hosts a version of electrodynamics where all charged objects are fermions.

Following the aforementioned examples, it is believed that such symmetry protected topological phases are characterized by their edge states, since they appear to be trivial in the bulk ${ }^{1}$. This definition implies that the physics which may arise at the edge of a $d+1$-dimensional $\mathrm{SPT}^{2}$ (and any low-energy effective field theory description thereof) must have features which may not arise intrinsically, in the absence of the extradimensional bulk. That is, there must not be a local lattice model (or other regulator) in strictly $d-1$ spatial dimensions which regulates the edge theory and preserves all of its symmetries. For example, there is no way to regulate a chiral edge mode in one dimension.

This realization $[6,7]$ implies that the study of SPT states may be used to identify obstructions to symmetric regulators of quantum field theory (QFT). In simple examples, such an obstruction can be identified with an 't Hooft anomaly coefficient [8], a well-known obstruction to gauging a global symmetry of a field theory. When realized at the edge, the bulk theory cancels the anomaly by anomaly inflow [9]. However, there are examples, particularly for discrete symmetries, where there is no previously-known anomaly $^{3}$. Examples of such obstructions which go beyond familiar global anomalies include many interesting states in $2+1$ dimensions, such as the algebraic vortex liquid [6], time-reversal-invariant $\mathbb{Z}_{2}$ gauge theory where all quasiparticles are fermions (the "all-fermion toric code") [6, 15], other topologically ordered states in $2+1$ dimensions [16-21], and a simple three dimensional example [7].

\footnotetext{
${ }^{1}$ The subject is reviewed in $[4,5]$.

${ }^{2}$ Following Sachdev's useful convention, we'll use $D=d+1$ to denote the number of spacetime dimensions.

${ }^{3}$ Formal attempts to interpret SPT obstructions in these terms include [10-14].
} 
This paper may be regarded as a sequel to [7], which identified an obstruction to a regulator for 'pure' $\mathrm{U}(1)$ gauge theory which manifestly preserves electromagnetic duality $^{4}$. While this is a gaussian model, such a no go result is interesting given attempts to construct such manifestly duality-symmetric realizations [23]. Further, it shows the impossibility of gauging electromagnetic duality, a conclusion which was argued from a very different point of view in [24-26].

Here we point out that a stronger obstruction may be found by adding 'matter' to the bulk model studied in [7]. The model we find at the surface is $3+1$-dimensional electrodynamics where all of the minimally-charged (electrically and/or magnetically) particles are fermions. This system has been discussed recently in [1], which demonstrated that it does not admit an interface with vacuum - it is not 'edgeable'. To be precise, ref. [1] showed that all-fermion electrodynamics cannot be realized in $3+1$ dimensions if the microscopic regulator consists entirely of bosonic degrees of freedom. If we add to the microscopic physics gauge-invariant fermion degrees of freedom, then we can bind the gauge invariant fermion to the minimally charged fermionic objects to produce minimally charged bosonic objects. Bosonic electrodynamics of course can be regulated in strict $3+1$-dimensions, by $\mathrm{U}(1)$ lattice gauge theory [27], or (more locally) by a $\mathrm{U}(1)$ toric code $[28,29]$.

This example is particularly dramatic because the obstruction arises in the absence of any symmetry (including translations). The $4+1$-dimensional phase is a short-rangeentangled state which is guaranteed to have an interesting edge no matter what horrors of disorder we subject it to. The only other known examples of this type are (copies of) the fermionic chiral $(p+i p)$ superfluid states ${ }^{5}$, Kitaev's $\mathrm{E}_{8}$ state of bosons [30-32] (both in $2+1$ dimensions), and Kitaev's majorana chain in $1+1$ dimensions [33] (provided we assume fermion number is unbreakable).

We note that the classification of [13] includes a nontrivial state in $4+1$ dimensions without symmetry. Ref. [34] attributes fermionic excitations to its surface states. We anticipate that the independent construction in this paper can be interpreted as a physics-based realization of the machinery in that work.

Why is the bulk nontrivial? The fact that the edge of the $4+1$-dimensional system realizes all-fermion electrodynamics, combined with an argument that all-fermion electrodynamics cannot be regulated in $3+1$ dimensions, implies that the bulk is a non-trivial 4+1-dimensional state of matter. Ref. [1] (Appendix D) has given one such argument for the absence of a $3+1 d$ regulator of all-fermion electrodynamics. Hence the bulk is a nontrivial state of matter, any representative groundstate of which is not adiabatically deformable to a product state. We provide two independent demonstra-

\footnotetext{
${ }^{4}$ The edge theory of this model was studied further in [22].

${ }^{5}$ in which the $\mathbb{Z}_{2}$ symmetry is ungauged and the vortices are not dynamical objects
} 
tions of bulk non-triviality, one from the point of view of the edge (in $\S 2.3$ ), and one that uses directly the bulk (in $\S 5$ ). Since no symmetry is required to define the bulk state, it is a topological phase of matter which is protected from all weak Hamiltonian perturbations. However, it is still short-range entangled [35,36]: two copies of the bulk state can be deformed into a product state, so it is its own 'inverse state'.

In the context of microscopic bosonic phases, the only other known example of a short-range entangled state which is distinct from the trivial phase in the absence of any symmetry is the $\mathrm{E}_{8}$ state in $D=2+1$ dimensions $^{6}$. As stated above, the distinguishing feature of the $E_{8}$ state is its chiral edge modes at an interface with the vacuum. A sharp and universal characterization of these chiral edge modes is the thermal Hall response: heat will be transported uni-directionally without dissipation along the boundary of the sample. In the language of anomalies, the nontriviality of the above $D=2+1$ example is demonstrated by the chiral central charge $c_{-} \equiv c_{L}-c_{R}$ of the edge states. $c_{-}$represents a gravitational anomaly of the edge CFT, and this is a construction of gravitational anomaly inflow.

In the $4+1$-dimensional example studied here, the analogous signature of the nontriviality of the state seems to be fermion number anomaly inflow, as we show in $\S 5$.

We demonstrate that this effect also occurs in the beyond-cohomology $D=3+1$ boson SPT protected by time reversal symmetry studied in $[6,15,17,37,38]^{7}$. A possible surface termination of this SPT consists of an all-fermion toric code, a model which has no $D=2+1$ realization with time reversal symmetry. Our claim implies that the preservation of time-reversal in the all-fermion toric code comes at the cost of the conservation of fermion number!

We emphasize that the main conclusion of this paper pertains to models made from bosons in $D=4+1$ dimensions. As we show, the addition of microscopic gaugeinvariant fermions to the system removes any obstruction to realizing the edge physics in strict $D=3+1$ dimensions. Such a gauge-invariant local fermion cannot arise at the edge of a bosonic system. From the point of view of a lattice field theorist attempting to regularize the given low-energy field theory, having to add an extra species of massive fermion at the cutoff may not seem like a huge price. However, we regard the demonstration that such a step is required as fascinating and requiring a systematic understanding.

The paper is structured as follows. First (§2), we review the physics of two-form

\footnotetext{
${ }^{6}$ as well as multiple copies of this state, which comprise an integer classification

${ }^{7} \mathrm{~A}$ related phenomenon was described for edge states of $3+1 \mathrm{~d}$ SPTs whose protecting group contains $\mathrm{U}(1)$ in [17]. In that case, the anomaly occurs upon gauging the $\mathrm{U}(1)$.
} 
Chern-Simons (" $B d C$ ") theory in $4+1$ dimensions and show that it admits an edge which supports all-fermion electrodynamics. The group of electromagnetic duality transformations, which can be realized as an exact symmetry of the bulk $B d C$ theory, plays an important role in the analysis. Second (§3), by considering the path integral of all-fermion electrodynamics on $\mathbb{C P}^{2}$, we show that all-fermion electrodynamics cannot have a bosonic regulator. This constitutes a proof of bulk non-triviality via edge non-regularizability. Third ( $\S 4)$, we show how to construct the bulk non-trivial state from layers of ordinary (e.g., with bosonic charges) electrodynamics by condensing dyon strings. Finally, we show how to interpret the obstruction in terms of a fermion number anomaly of the all-fermion electrodynamics $(\S 5)$ and show that similar physics is realized in the non-trivial time-reversal $(\mathcal{T})$ protected bosonic SPT in $3+1$ dimensions $(\S 6)$.

\section{The $B d C$ model coupled to matter}

\section{$2.1 \quad B d C$ summary}

We begin by describing the action of the $B d C$ theory and reviewing its basic properties [39-50]. Consider 2-forms $B_{M N}^{I}\left(I=1 . . N_{B}\right.$ labels the form, $M N$ are the spacetime indices) in $4+1$ dimensions, with the topological action

$$
S[B]=\frac{K_{I J}}{2 \pi} \int_{\mathbb{R} \times \Sigma} B^{I} \wedge \mathrm{d} B^{J}
$$

where $\Sigma$ denotes the space of interest.

To process this action, we need a little exterior algebra: a $p$-form $\alpha_{p}$ and a $q$-form $\beta_{q}$ satisfy $\alpha_{p} \wedge \beta_{q}=(-1)^{p q} \beta_{q} \wedge \alpha_{p}$ and $\mathrm{d}\left(\alpha_{p} \wedge \beta_{q}\right)=\mathrm{d} \alpha_{p} \wedge \beta_{q}+(-1)^{p} \alpha_{p} \wedge \mathrm{d} \beta_{q}$. Hence we have $B^{I} \wedge B^{J}=B^{J} \wedge B^{I}$ and

$$
B^{I} \wedge \mathrm{d} B^{J}=\mathrm{d}\left(B^{I} \wedge B^{J}\right)-\mathrm{d} B^{I} \wedge B^{J},
$$

so up to a total derivative the action is anti-symmetric in $I J$. Thus $K$ is an antisymmetric $2 N_{B} \times 2 N_{B}$ matrix. Shortly we show that in order for (1) to govern the low-energy effective field theory of a short-range entangled bulk state, $K$ must also be an integer matrix with $\operatorname{det}(K)=1$. Also, since $B \wedge \mathrm{d} B=\frac{1}{2} \mathrm{~d}(B \wedge B)$ is a total derivative, we must have an even number of such two-forms. To see that there must be an even number, note that we view the topological field theory action (1) as the extreme low-energy effective field theory for a gapped state of matter; this is only self-consistent if it is stable to perturbations by generic irrelevant bulk terms involving 
these low-energy degrees of freedom. If there were an odd number of two-forms, the addition of generic irrelevant bulk terms - in particular the bulk Maxwell term

$$
\frac{1}{g^{2}} \int_{\mathbb{R} \times \Sigma} d B \wedge \star d B
$$

(where $\star$ is the Hodge duality operation) - would produce a propagating gapless photon in $D=4+1$.

The local gauge transformations $B^{I} \simeq B^{I}+d \lambda^{I}$ are redundancies of the model. An important further ingredient of the definition of the model $[48,50,51]$ is the 'large gauge' identifications:

$$
B^{I} \simeq B^{I}+n^{\alpha} \omega_{\alpha}, \quad\left[\omega^{\alpha}\right] \in H^{2}(\Sigma, \mathbb{Z}), \quad n^{\alpha} \in \mathbb{Z}^{b^{2}(\Sigma)}
$$

where the betti number $b^{2}(\Sigma) \equiv \operatorname{dim} H^{2}(\Sigma, \mathbb{Z})$ is the dimension of the second integer cohomology of $\Sigma$. This requires the entries of $K$ to be integers ${ }^{8}$.

The equations of motion following from (1) are, $\forall I$,

$$
K_{I J} d B^{J}=0
$$

When $K$ has full rank, these equations are solved by flat two-form fields, which are identified by local gauge equivalences, and there are therefore no local degrees of freedom. As a result, the gauge-inequivalent operators (analogs of Wilson loop operators) are labelled by cohomology classes

$$
\mathcal{F}_{\omega}(m) \equiv e^{2 \pi \mathbf{i} m_{I} \int_{\omega} B^{I}}
$$

with $[\omega] \in H^{2}(\Sigma, \mathbb{Z})$. The identification (4) on $B$ implies $m^{I} \in \mathbb{Z}$.

Using equal-time canonical commutators for $B^{I}$, the flux operators (6) satisfy a Heisenberg algebra:

$$
\mathcal{F}_{\omega_{\alpha}}(m) \mathcal{F}_{\omega_{\beta}}\left(m^{\prime}\right)=\mathcal{F}_{\omega_{\beta}}\left(m^{\prime}\right) \mathcal{F}_{\omega_{\alpha}}(m) e^{2 \pi \mathbf{i} m_{I}^{\alpha} m_{J}^{\prime \beta}\left(K^{-1}\right)^{I J} \mathcal{I}_{\alpha \beta}}
$$

Here

$$
\mathcal{I}_{\alpha \beta} \equiv \int_{\Sigma} \omega_{\alpha} \wedge \omega_{\beta}
$$

is the intersection form on $H^{2}(\Sigma, \mathbb{Z})$, which is a $b^{2}(\Sigma) \times b^{2}(\Sigma)$ symmetric matrix. Consider the minimal case (relevant later on) where $\Sigma=\mathbb{C P}^{2}$, for which $b^{2}\left(\mathbb{C P}^{2}\right)=1$ and $\mathcal{I}=1$. The smallest representation of the algebra $(7)$ is then $|\operatorname{Pf}(K)|$-dimensional,

\footnotetext{
${ }^{8}$ In this paper we will only discuss this model on manifolds without torsion homology. For the machinery required to lift this restriction, see [52].
} 
which must be unity for short-range-entangled states. Hence we require $\operatorname{det} K=$ $\operatorname{Pf}^{2}(K)=1$.

The $B d C$ theory is a special case of (1) where we take $N_{B}=2$ and let $B^{1}=B$, $B^{2}=C$, and $K=k i \sigma^{y}$; we must set $k=1$ for this state to be short-range entangled (when $k>1$ the system has topological ground state degeneracy depending on $b^{2}(\Sigma)$, namely $k^{b^{2}(\Sigma)}$ groundstates). We now review its physics on a space with boundary $[7,22]$. In the presence of a boundary, the solutions of the equations of motion produce physical excitations: a one-form field $a$ localized at the boundary. This mode is physical because gauge transformations which are nontrivial at the boundary do not preserve (1). Boundary terms (whose coefficients are non-universal) produce the Maxwell action for $a$. In particular, the boundary condition arising from variation of an action with the leading irrelevant operators (i.e. the bulk Maxwell terms (3)) is:

$$
\left.\left(\frac{k}{2 \pi} B-\frac{1}{2 g^{2}} \star_{4} C\right)\right|_{\partial \Sigma_{4}}=0 .
$$

Upon a convenient rescaling, the identification of boundary degrees of freedom is:

$$
B=d a, \quad C=\star d a .
$$

An important symmetry of the topological action (1) is the group $\mathrm{SL}\left(2 N_{B}, \mathbb{Z}\right)$ of field redefinitions that preserve the identifications (4). We emphasize that this symmetry is not necessary for the $4+1$ bulk to be distinct from a trivial phase; indeed, this symmetry may be broken by UV physics, but it turns out to be very convenient to analyze certain topological features of the physics assuming this symmetry holds. In the case of the $B d C$ theory, the group is $\mathrm{SL}(2, \mathbb{Z})$ and it is closely related to the group of duality transformations on the boundary electrodynamics.

The action of $\mathrm{SL}(2, \mathbb{Z})$ on $B, C$ is in the fundamental representation

$$
\left(\begin{array}{l}
B \\
C
\end{array}\right) \rightarrow \mathrm{M}\left(\begin{array}{l}
B \\
C
\end{array}\right)
$$

with $\mathrm{M} \in \mathrm{SL}(2, \mathbb{Z})$. The ' $\mathrm{T}$ ' transformation $\mathrm{T}=\left(\begin{array}{ll}1 & 1 \\ 0 & 1\end{array}\right)$ is a symmetry because $B \wedge d B$ is a total derivative; by (8), this transformation shifts the theta angle of the surface gauge theory by $2 \pi$. The ' $S$ ' transformation $S=\left(\begin{array}{rr}0 & 1 \\ -1 & 0\end{array}\right)$ is a symmetry because of (2), and acts as electromagnetic duality on the boundary gauge field. These two transformations generate $\mathrm{SL}(2, \mathbb{Z})$. Notice that on $B, C$, the $\mathbb{Z}_{2}$ center of the duality group acts nontrivially (this is charge conjugation at the edge). 


\subsection{Coupling to strings (matter)}

Just as a one-form gauge field $A$ couples minimally to the worldline of a charge, $\int_{\text {worldline }} A$, a two-form gauge field $B$ couples minimally to the worldsheet of a string, $\int_{\text {worldsheet }} B$. Adding matter to Chern-Simons theory is usually [53] described in terms of a statistics vector, $l_{I}$, so that the quasiparticle (here, 'quasistring') current is the two-form $l_{I} \star d B^{I}$. If $B^{I}$ are normalized as in (1), the $l_{I}$ must be integers, so that $e^{\mathrm{i} \oint_{\Sigma} l_{I} B^{I}}$ is periodic under shifts of the periods of $B^{I}$ over all topologically nontrivial 2-cycles $\Sigma$.

Gauge invariance under $B^{I} \simeq B^{I}+d \lambda^{I}$ requires that strings not end in the bulk of the sample. However, strings can end at an interface with vacuuum. Then because of the identification (8), the ends of the strings are electric and magnetic charges under the boundary gauge field $a$. Indeed, given a string which terminates at a boundary, the coupling $\int_{\text {worldsheet }} B$ reduces to the coupling $\int_{\text {worldline }} a$ by Stokes' theorem.

We discuss in detail below the statistics of the surface particles arising at the ends of the bulk string matter. As a preliminary, note that the modular group $\operatorname{SL}(2, \mathbb{Z})$ acts on the string matter as well. This action is necessary to preserve the coupling between string worldsheets and two-form fields.

\section{$2.3 \quad$ Edge physics}

We now consider an edge of the $D=4+1$ dimensional $B d C$ bulk which supports $\mathrm{U}(1)$ electrodynamics in $D=3+1$ dimensions [7]. As anticipated in the introduction, the crucial question is: what are the statistics of the basic charged particles on the edge. Because the edge electrodynamics is a stable phase of matter and because the statistics of the charged particles is topological data, these statistics must be stable to the breaking of all symmetries in the problem. Hence to determine the statistics we may assume extra symmetry and be confident that we have the correct statistics even if we later break the symmetry (for example by allowing the electron and monopole to have different masses) to realize the generic situation.

Thus suppose that we preserve the manifest $\mathrm{SL}(2, \mathbb{Z})$ duality symmetry of the $B d C$ theory. Duality symmetry implies that the charge $e$ and the monopole $m$ have the same statistics, since they are related by the symmetry. For $\mathrm{G}=\mathrm{U}(1)$, the full duality group

is $\mathrm{SL}(2, \mathbb{Z})$, and it acts on the charge vector by $\left(\begin{array}{c}q_{e} \\ q_{m}\end{array}\right) \rightarrow \underbrace{\left(\begin{array}{ll}a & b \\ c & d\end{array}\right)}_{\in \mathrm{SL}(2, \mathbb{Z})}\left(\begin{array}{c}q_{e} \\ q_{m}\end{array}\right)$. In particular,

the transformation $\left(\mathrm{T}^{\mathrm{t}} \mathrm{S}\right)^{-1}$ takes the charge to the $(1,1)$ dyon $\epsilon \equiv \mathrm{em}$. The boundstate with these quantum numbers must therefore have the same statistics as the charge and 
the monopole. Since these are particles in $3+1$ dimensions, they may be either all bosons or all fermions.

Naively both possibilities are allowed, but in fact, if $e$ and $m$ have the same statistics, then $\epsilon$ must be a fermion. This phenomenon is sometimes called 'spin from isospin' $[54,55]$ (when the electrodynamics is UV completed by SU(2) gauge theory with an adjoint higgs field). Note that we must assume there are no gauge-invariant fermions around, otherwise we could bind such a fermion to the dyon without changing its charges and turn it into a boson.

To see this efficiently, consider two identical dyons wellseparated in space compared to any cutoff scales. Since they are identical particles, moving one of them adiabatically in an arc of angle $\pi$ around the other results in the

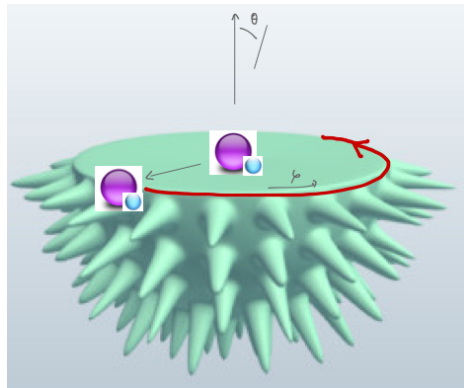

Figure 1: A depiction of the calculation of dyon statistics. The spikes represent the flux produced by the dyon at the center. same state (up to an innocuous center-of-mass translation). The Berry phase acquired in doing so is

$$
\varphi=e \int_{0}^{\pi} d \varphi \underbrace{\mathcal{A}_{\varphi}\left(\theta=\frac{\pi}{2}, \varphi\right)}_{\text {Dirac monopole field }} \stackrel{\text { Dirac }}{=} \pi g e .
$$

If $g$ and $e$ have the minimal charges, saturating the Dirac quantization condition, then

$$
\psi\left(x_{1}, x_{2}\right)=e^{\mathbf{i} \varphi} \psi\left(x_{2}, x_{1}\right)=-\psi\left(x_{2}, x_{1}\right)
$$

and these particles are fermions. The extra $\frac{\hbar}{2}$ unit of angular momentum comes from the electromagnetic field. Note that any exchange phase coming from the constituent $e$ and $m$ particles cancels because we assumed they were both bosons or both fermions.

Thus we reach the remarkable conclusion that the model with a duality-symmetric spectrum of all bosons is not even self-consistent! On the other hand, an all-fermion spectrum is self-consistent: because of the additional $\frac{\hbar}{2}$ unit of angular momentum in the electromagnetic fields, the dyon boundstate of two fermions is still a fermion [56].

To prove that the bulk is non-trivial we argue by contradiction and suppose that allfermion electrodynamics can be realized in strict $D=3+1$ dimensions with microscopic bosons only. Then we could place a field theory realization on $\mathbb{C P}^{2}$ since the theory is bosonic and requires no spin structure for its definition. However, something bad happens, which we describe next, in $\S 3$.

Hence there must be no UV completion in the same dimension with only microscopic bosons. Since the BdC theory provides a UV completion of all-fermion electrodynamics with only bosons at its edge, it follows that the bulk $B d C$ phase is necessarily distinct 
from the trivial phase. Alternatively, the results of [1] also imply that all-fermion electrodynamics cannot be realized in strict $D=3+1$ dimensions without gaugeinvariant fermions, so again we conclude that the bulk $B d C$ phase is distinct from the trivial phase.

\section{The Bad Thing that Happens on $\mathbb{C P}^{2}$}

To show the impossibility of a bosonic regulator of all-fermion QED, we show that there is no consistent way to define the partition function on $\mathbb{C P}^{2}$. To make the argument we suppose:

Postulate 1: A U(1) gauge theory with gapped matter (and hence the value of the $\mathrm{U}(1)$ gauge theory path integral on a closed manifold $\mathrm{M}$, modulo non-universal garbage) is specified by the theta angle and the coupling and by the spectrum of charges.

But what theta angle and coupling you ask? What data about the spectrum? More specifically, we suppose:

Improved Postulate 1: The value of the gauge theory path integral on a closed manifold $M$, modulo non-universal garbage, depends only on the bare coupling $\tau=$ $\theta+\frac{4 \pi \mathbf{i}}{g^{2}}$ (and $\bar{\tau}$ ), and on a choice of statistics for the excitations with minimal electric and magnetic charges, $e, m$. We include the dependence on the particle masses and various other couplings in the category of 'non-universal garbage'.

A crucial point here is that the effective theta angle (at energies below the gap to charged excitations) may receive contributions from integrating out the matter, as is familiar from the study of topological insulators (e.g. [57,58]). A useful perspective then, is that all such gauge theories may be realized by coupling "pure" U(1) gauge theory to bosonic or fermionic matter in various $\mathrm{U}(1)$ SPTs, that is: gauging the $\mathrm{U}(1)$ global symmetry. A possibility which we must also discuss is a case with no charged matter, studied with related intent in $[7,59]$.

Let us consider the action of duality on the gauge theory partition function. We are free to relabel the gauge fields using the electric-magnetic duality group $\tau \rightarrow \frac{a \tau+b}{c \tau+d}$, $(a, b, c, d \in \mathbb{Z}, a d-b c=1)$ but we must keep track of the particle statistics as well. We will be most interested in the $\mathrm{T}$ transformation which takes $\theta \rightarrow \theta+2 \pi$. Recall [56] that shifting the theta angle produces a spectral flow on the charge lattice: monopoles acquire electric charge proportional to $\frac{\theta}{2 \pi}$.

Therefore (in the absence of other data, an absence for which we argue below) the choice of statistics of the charged matter gives an invariant meaning to the duality frame. Denote the statistics labels on the gauge theory as follows: BBF if $e$ is a boson, $m$ is a boson and (therefore) $e m$ is a fermion, BFB if $e$ is a boson, $m$ is a fermion, $e m$ is 
a boson, etc. Note that by the spin-from-isospin argument, this labeling is redundant (the statistics of $e m$ is determined by those of $e$ and $m$ ), but it will help emphasize the important distinction between the all-fermion case and the other cases. If we allow neutral fermions, then we have both bosons and fermions in each charge sector, and the labeling scheme breaks down; we assume no neutral fermions. If there are no charged particles, then any duality transformation in $\operatorname{SL}(2, \mathbb{Z})$ is a redundancy: a relabeling of fields.

For example, the Witten effect [56] implies that for any four-manifold $M$,

$$
\mathrm{\top}: Z_{M}(\tau, B B F)=Z_{M}(\tau+1, B F B)
$$

On the other hand, consider the case where $M=\mathbb{C P}^{2}$; this example is interesting because it has a two-cycle $h$ with unit self-intersection. This means that a line bundle with $\mathbf{c}_{1}=h$ has

$$
\frac{1}{4 \pi^{2}} \int_{\mathbb{C P}^{2}} F \wedge F=1
$$

Therefore the partition sum is

$$
Z_{\mathbb{C P}^{2}}(\theta)=\int D A e^{-S_{0}[A]+i \frac{\theta}{8 \pi^{2}} \int_{\mathbb{C P}^{2}} F \wedge F}=\sum_{\mathbf{c}_{1}=n h} \int_{C_{n}}[D A]_{n} e^{-S_{0}[A]+i \frac{\theta}{2} n}
$$

where $C_{n}$ labels the sector of the gauge field configuration space with $\int_{h} \frac{F}{2 \pi}=n . Z_{\mathbb{C P}^{2}}(\theta)$ is therefore periodic in $\theta$ with period $4 \pi$. (This fact is discussed in detail in [59]; the odd intersection form on $\mathbb{C P}^{2}$ also plays a role in the discussion of [13].)

Since we know that $Z_{\mathbb{C P}^{2}}(\tau, B B F)$ is not the same as $Z_{\mathbb{C P}^{2}}(\tau+1, B B F)$ but that it is the same as $Z_{\mathbb{C P}^{2}}(\tau+2, B B F)$, it follows that integrating out charged matter which makes the monopole a fermion generates an extra theta term with coefficient $2 \pi(\bmod$ $4 \pi)$, in agreement with previous results [56].

Finally, let us turn to the case of $Z(\tau, F F F)$. By the Improved Postulate 1 we have

$$
Z_{M}(\tau, F F F)=Z_{M}(\tau+1, F F F)
$$

for all 4-manifolds $M$ on which the theory is defined. However, this equation can only be true if $M$ has an even intersection form. If the theory had a bosonic regulator, then we could place it on manifolds with an odd intersection form and no spin structure, such as $\mathbb{C P}^{2}$. The theory cannot be placed on manifolds with odd intersection form, hence the theory does not have a bosonic regulator ${ }^{9}$.

\footnotetext{
${ }^{9}$ Note than an additional consequence of its lack of spin structure is that $\mathbb{C P}^{2}$ cannot occur as the boundary of some smooth, compact 5-manifold; it has a non-vanishing Stiefel-Whitney number. See Theorem 4.10 of [60]. This theorem prevents a contradiction with the fact that the partition function
} 
In order for this periodicity in $\theta$

$$
Z_{\mathbb{C P}^{2}}(\tau+1, F F F) \stackrel{!}{=} Z_{\mathbb{C P}^{2}}(\tau, F F F)
$$

to be a consistency condition (that is: its violation is a gauge anomaly) we require that the modular properties of the partition function are determined entirely by the spectrum of electric and magnetic charges. We argue for this claim in a series of comments, which can be regarded as an attempt to make precise the lack of structure in $\mathrm{U}(1)$ gauge theory:

- First, we emphasize that the statistics of particles in all charge sectors $\left(q_{e}, q_{m}\right)$ are fixed by the elementary ones $(1,0),(0,1)$ (the generators of the charge lattice) and the demand that there are no neutral fermions. For example, the spectrum of the FFF theory cannot contain a magnetic-charge-two monopole which is a fermion, because then binding such an object to the (boson) boundstate of two charge (-1) monopoles would produce a neutral fermion.

- In gauge theories with more interesting gauge group or massless matter content, other labels are required to specify the partition function. For example, gauge theories where a $2 \pi$-shift of $\theta$ produces a different gauge theory were discussed recently in [61]. The new labels there arise from extra topological invariants (beyond the Pontryagin invariant) of gauge bundles whose structure group (the gauge group) is semisimple but not simply connected (a pedagogical exposition of this subject can be found in $\S 3$ of [62]).

- Here we are studying $\mathrm{G}=\mathrm{U}(1)$ where this issue does not arise. That is: The smooth topological data of a line bundle (the structure group is $\mathrm{U}(1)$ ) on a simply connected manifold is just the first Chern class (for a discussion which makes this clear see e.g. page 3 of [59]). Therefore this possibility for modifying the periodicity of theta is not available.

- Another potential source of a theta-dependent phase in the partition function is a possible $\tau$-dependence in the gravitational couplings in the effective action for the gauge fields upon integrating out the gapped charged matter. Such couplings are crucial in computing the partition function of topologically twisted gauge theories [62] on various four-manifolds, and are discussed further in [59]. In that context, such terms produce anomalous factors under the $\mathrm{S}$ transformation, but not under the $\mathrm{T}$ transformation.

of the all-fermion electrodynamics on $M$ can be obtained from the $B d C$ theory on a space whose boundary is $M$. Two disjoint copies of $\mathbb{C P}^{2}$ can occur as the boundary of e.g. $\mathbb{C P}^{2} \times[0,1]$. In this case, the instanton sums in the two copies of all-fermion electrodynamics are correlated by the fact that $B=d a_{1}+d a_{2}$ is flat in the bulk, again avoiding contradiction. 
Further, to see that this is not a meaningful loophole here, we can take the perspective described above: we couple an SPT with $G=U(1)$ symmetry (in curved space) to the electromagnetic field. The gravitational effective action for the SPT is completely fixed before the coupling to the EM fields, which is when $\tau$ is introduced. Therefore, the $\tau$ dependence of the action below the gap is completely fixed by the matter content.

So the basic question is: what other kinds of UV gerbils can there be in $\mathrm{U}(1)$ gauge theory which might affect the $\tau$-dependence of the partition function? We can see that the answer is 'none' as follows.

- Adding fermions restores $2 \pi$ periodicity of the theta angle. This matches nicely with the fact $[63,64]$ that the $\theta$ angle for a background gauge field is only periodic $\bmod 4 \pi$ in a system made of bosons (since the surface at $\theta=2 \pi$ would have oddinteger quantum Hall response, which is not compatible with bosonic statistics of all neutral excitations). This argument implies that only fermions in the charge spectrum can change the periodicity in theta by $2 \pi$. But we've already accounted for the fermionic charges.

- As a nice corroboration of our understanding, note that the counting of non-trivial states here is consistent with the counting of SPT states [64]. In particular, absent time reversal, the three states $\mathrm{BBF}, \mathrm{FBB}$, and $\mathrm{BFB}$ are smoothly connected.

- Finally, we believe that the argument described here implies that there is no such thing as 'pure' $U(1)$ gauge theory, i.e. $\mathrm{U}(1)$ gauge theory without any charged matter at all. From the low energy point of view, the problem with the allfermion model is simply that the spectrum is duality invariant, and so cannot be rearranged by the Witten effect. The same is true if there are no charges, and so we have:

$$
Z_{M}(\tau+1,---) \stackrel{!}{=} Z_{M}(\tau,---)
$$

(where the dashes emphasize the absence of charged matter). The fact that this demand is violated for $M=\mathbb{C P}^{2}$ was observed in [59]. We believe that the above argument implies that this failure should be regarded as an inconsistency. We note that there is no known regulator of this model. The $\mathrm{U}(1)$ toric code is described at low energies by electromagnetism coupled to gapped matter with spectrum BBF. Ordinary lattice gauge theory is simply the limit of the toric code where the electric excitations are made infinitely heavy; in particular it still contains gapped magnetic monopole excitations. (A term by which one might try to lift these excitations completely, e.g. $\sum_{\text {plaquettes }} \Delta \cdot(\Delta \times a)$, is not single-valued under the equivalence $a_{\ell} \rightarrow a_{\ell}+2 \pi n_{\ell}, n_{\ell} \in \mathbb{Z}$.) 
Perhaps there exists a consistent low energy theory where there are only magnetic charges; in that case, we have the condition

$$
Z_{M}(\tau+1,-B-) \stackrel{!}{=} Z_{M}(\tau,--B)
$$

which is not falsified by the lack of a spin structure of $M$.

The fact that there is an obstruction to a duality-invariant regulator of 'pure' electromagnetism was argued in [7] (with hindsight, this result also follows from the calculation of [59]). Here we are making the further claim that there is no regulator at all. The argument above shows that there is no bosonic regulator. Many of the other anomalies discussed in this paper may be cured by adding neutral fermions. In this case, it is difficult to see how the addition of gapped, neutral fermionic excitations can help. In particular, the fact that the fermion is neutral means that integrating it out does not generate a theta term. However, the presence of microscopic neutral fermions amounts to a refusal to put the system on a manifold without spin structure, such as $\mathbb{C P}^{2}$ ! (Since the fermions are neutral, the existence of a $\operatorname{spin}_{c}$ structure does not help.) So indeed there is no obstruction to a fermionic regulator.

We discuss below in $\S 6$ the consequences of the analogous line of argument for the all-fermion toric code in $D=2+1$.

\section{Coupled Layer Construction}

In this section, we describe a $4+1 \mathrm{~d}$ local lattice model which realizes the continuum model above, using a coupled layer construction (precedents for such an approach include [6,31,65-67]). Like the edge-based proof of bulk non-triviality, the motivation for the layer construction comes from edge physics. If SPTs are only non-trivial because of their edge states, then we should be able to construct interesting SPTs by sewing together pairs of edge states as follows.

First, observe that every short-range entangled state with a non-trivial edge has an inverse short-range entangled state (obtained be reversing the orientation) with a non-trivial edge and with the property that the composite short-range entangled state has a trivial edge. In other words, for every non-trivial (anomalous) edge $\mathcal{E}$ there is another non-trivial edge $\mathcal{E}^{-1}$ such that $\mathcal{E} \times \mathcal{E}^{-1} \sim 1$ is trivial. We then imagine a stack of such edges, $\left(\mathcal{E}_{1} \mathcal{E}_{1}^{-1}\right) \ldots\left(\mathcal{E}_{n} \mathcal{E}_{n}^{-1}\right)$, which can clearly be reduced to a trivial state by

pairing $\mathcal{E}_{i}$ with $\mathcal{E}_{i}^{-1}$. However, we may also pair $\mathcal{E}_{i}^{-1}$ with $\mathcal{E}_{i+1}$ in such a way that the edges $\mathcal{E}_{1}$ and $\mathcal{E}_{n}^{-1}$ are left un-paired. Assuming interactions are local in the layer index 
$n$, these remaining actual edge states cannot be paired with each other and we have produced a non-trivial bulk state. More generally, we may take any lower dimensional "layers" and try to couple them in a similar non-integrable fashion to produce a bulk short-range entangled state with non-trivial edge states.

We make a coupled-layer construction of the all-fermion electrodynamics following (very directly) the one made in [6] for the all-fermion toric code. It produces a trivial bosonic bulk, and the correct edge physics. As an essential part of the construction, we are able to argue that this bosonic bulk is well-described by the $B d C$ theory.

The method by which we construct the bulk can be called 'dyon string condensation'. It has a lot in common with the dyon condensation mechanism of statistics transmutation in $3+1$ dimensions employed in [68]. The construction can also be regarded as an oblique version of 'deconstruction' of the extra dimension [69]; this will be a useful perspective for understanding the origin of the $B \wedge d C$ term.

First we give a brief summary of the construction:

- Each layer, labelled $i=1 . . n$, is ordinary electrodyam-

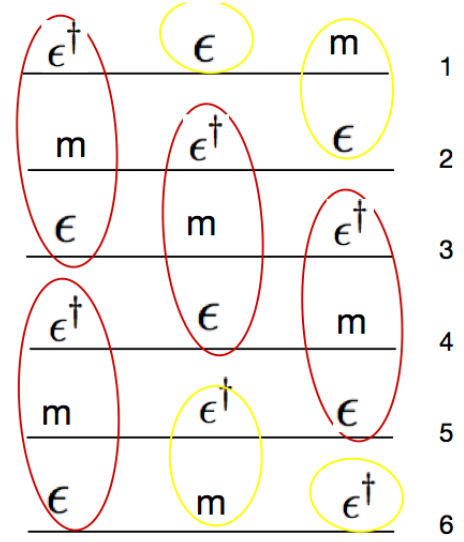

Figure 2: A representation of the coupled layer construction, following [6]. The layers are coupled by condensing the objects circled in red.

ics with bosonic charges: the electron and monopole $e_{i}, m_{i}$ are gapped bosons. This model is certainly regularizable in $3+1 \mathrm{~d}$ by itself on an ordinary Hilbert space of bosons on links and sites. Denote the (fermionic) dyon in each layer as $\epsilon_{i}$.

- $b_{i} \equiv \epsilon_{i}^{\dagger} m_{i+1} \epsilon_{i+2}$ are mutually-local bosons.

- Condensing $b_{i}$ (obliquely) confines $a_{i+1}, i+1=2 \ldots N-1$.

- At the top layer: $m_{1} \epsilon_{2}, \epsilon_{1}^{\dagger} m_{1} \epsilon_{2}, \epsilon_{1}^{\dagger}$ survive, are fermions, and are the electron, monopole and dyon of a surviving (Coulomb-phase) U(1) gauge field. A similar statement pertains to the bottom layer.

In the bulk, in the continuum, we will arrive at the claim that this is the $B d C$ theory with gapped string matter.

\subsection{Warmup: deconstruction of lattice electrodynamics}

First consider the following toy example, which really is 'deconstruction' of $4+1 \mathrm{~d}$ $\mathrm{U}(1) \times \mathrm{U}(1)$ gauge theory on an interval, in the sense of [69]. (A quiver diagram for this construction, more familiar in the high-energy theory literature, appears in Fig. 3.) 
Collocate an even number $N$ of layers of (cubic, say) $3 d$ lattices each of which hosts $\mathrm{U}(1)$ lattice gauge theory coupled to charge-1 lattice bosons $e_{i}$, with arbitrary hopping terms in the three spatial dimensions. For definiteness, we could consider each layer in the zero-correlation length limit where it is described by a solvable Kitaev-like model with a rotor on each (oriented) link, $\left[\mathrm{E}_{l}, \mathbf{a}_{l^{\prime}}\right]=\mathbf{i} \delta_{l, l^{\prime}}, \mathrm{E} \in \mathbb{Z}, \mathbf{a} \simeq \mathbf{a}+2 \pi$, with

$$
\mathbf{H}_{\text {layer }}=\sum_{+}(\Delta \cdot E)^{2}-\sum_{\square} \cos (\Delta \times \mathbf{a}) .
$$

$\Delta$ is a lattice gradient operator. The charged bosonic matter arises at sites where $0 \neq \Delta \cdot \mathrm{E} \in \mathbb{Z}$.

Couple together the layers by the (completely local and gauge invariant) terms

$$
\Delta H=V \sum_{x} \sum_{i}\left(\left|\tilde{\mathbf{b}}_{i}(x)\right|^{2}-v^{2}\right)^{2} .
$$

Here $x$ labels a site of the 3 d lattice. Fig. 3 shows the case of $N=6$ layers, with $\tilde{\mathbf{b}}_{i}, i=1 . .4$ circled. Minimizing the potential (9) causes $\tilde{\mathbf{b}}_{i}$ to condense,

$$
\tilde{\mathbf{b}}_{i} \equiv e_{i}^{\dagger} e_{i+2}=v e^{\mathbf{i} a_{i, i+2}},
$$

higgsing $\prod_{i} \mathrm{U}(1)_{i} \rightarrow \mathrm{U}(1)_{\text {even }} \times \mathrm{U}(1)_{\text {odd }}$. The phases $a_{i, i+2}$ provide the link variables in the extra dimension. Layers with odd $i$ and even $i$ are decoupled. The result is $4+1 d$ Maxwell theory with $\mathrm{G}=\mathrm{U}(1)_{\text {even }} \times \mathrm{U}(1)_{\text {odd }}$, with massless bulk photons. So this is not the bulk state we are looking for, but it will be instructive.

$\mathrm{U}(1)$ lattice theory in $4+1$ dimensions should have a kinetic term for the link variables along the extra dimension. This $\mathrm{E}_{x, x+\hat{4}}^{2}$ term arises as follows. The conjugate variable $\mathrm{E}$ to $\mathbf{a}$ arises from the amplitude fluctuations of $\tilde{b}$ :

$$
\begin{gathered}
\tilde{\mathbf{b}}_{l}=e^{\mathbf{i} a_{l}}\left(v+\mathrm{E}_{l}\right), \quad \tilde{\mathbf{b}}_{l}^{\dagger}=\left(v+\mathrm{E}_{l}\right) e^{-\mathbf{i} a_{l}} . \\
{\left[\tilde{\mathbf{b}}_{i}^{\dagger}(x), \tilde{\mathbf{b}}_{j}(y)\right]=-\mathbf{i} \delta_{x y} \delta_{i j} \Longrightarrow\left[\mathbf{a}_{l}, \mathrm{E}_{l^{\prime}}\right]=-\mathbf{i} \delta_{l l^{\prime}} .}
\end{gathered}
$$

Expanding the condenser term (9) about the minimum, $\tilde{\mathbf{b}}^{\dagger} \tilde{\mathbf{b}}-v^{2}=2 v \mathrm{E}+. .$, we find

$$
\Delta H=V 4 v^{2} \sum_{l} \mathrm{E}_{l}^{2}+. .
$$
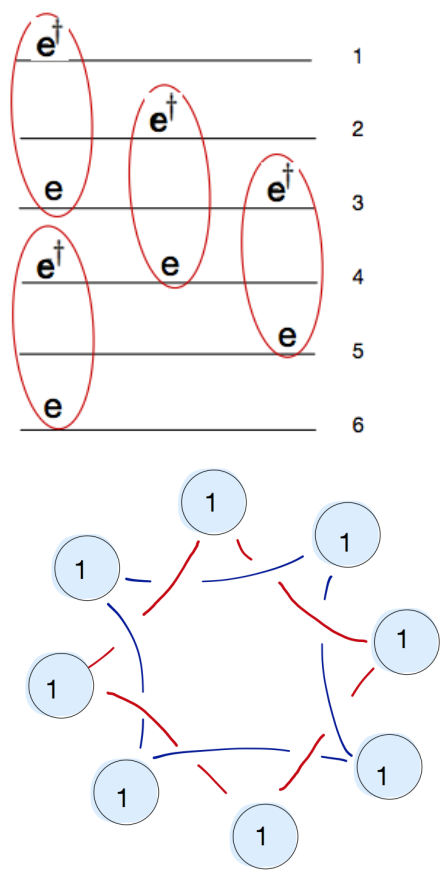

Figure 3: Two representations of the (warmup) coupled-layer construction for $D=4+1$ Maxwell theory with gauge group $\mathrm{U}(1)_{o} \times \mathrm{U}(1)_{e} . \quad$ The top figure is the direct analog of the previous figure; the bottom is a 'quiver' or 'moose' diagram familiar from the high energy physics literature. 
The Hamiltonian should also contain terms which suppress flux through plaquettes parallel to the extra dimension: $\sum_{\text {plaquettes } \| x^{4}} \cos \Delta \times \mathbf{a}$. These terms arise from microscopic gauge invariant terms including the hopping term for $\tilde{\mathbf{b}}$ :

$$
\Delta_{2} H=-V_{2} \sum_{x, i} \sum_{\hat{\mu} \neq \hat{4}}\left(\begin{array}{c}
\tilde{\mathbf{b}}_{i}(x+\hat{\mu}) e^{\mathbf{i} \int_{x}^{x+\hat{\mu}} \mathbf{a}_{i}-\mathbf{i} \int_{x}^{x+\hat{\mu}} \mathbf{a}_{i+2} \tilde{\mathbf{b}}_{i}^{\dagger}(x)}+h . c .
\end{array}\right)
$$

where $\mathbf{a}_{i}$ is the pre-existing gauge field within layer $i$. Upon condensing the $\tilde{\mathbf{b}}_{i}$, the new interlayer gauge field $\mathbf{a}_{i, i+2}$ combines with the existing within-layer gauge fields to form a closed Wilson loop in the $\mu 4$ plane for each term in the $\mu$ sum.

It will be useful to remind ourselves about magnetic monopoles in $\mathrm{U}(1)$ lattice gauge theory (e.g. [70]). A region $R$ of the lattice whose boundary $\partial R$ has $\oint_{\partial R} B=2 \pi g$ contains $g$ magnetic monopoles, $g \in \mathbb{Z}$. This means that the number of monopoles is not conserved on the lattice; for example, consider a region which is a single 3-cell $V$ of the lattice; we may change $\oint_{\partial V} B$ from 0 to $2 \pi$ without changing anything, since the gauge field is periodic $\mathbf{a} \simeq \mathbf{a}+2 \pi$ and $B=\vec{\nabla} \times \mathbf{a}$.

To make contact with the $B d C$ theory, it will be illuminating to dualize the odd/even gauge fields $a^{o / e}$ to 2 -form potentials: $f^{o / e}=d a^{o / e}=\star d C^{o / e}$. The action is

$$
S=\sum_{\alpha=o, e} \int_{5 d}\left(\frac{1}{g_{\alpha}^{2}} d C^{\alpha} \wedge \star d C^{\alpha}+C^{\alpha} \wedge \star j_{m}^{\alpha}\right) .
$$

By the Meissner effect, magnetic flux tubes of the broken relative $\mathrm{U}(1) \mathrm{s}$ collimate the monopoles into monopole strings. They must do so, since, by construction, objects magnetically charged under $a^{e / o}$ are minimally coupled to the dual field $C^{e / o}$ and must be strings. States where the total magnetic charge in different layers is not equal do not have finite energy. We sequester a few more details about this to appendix $\S \mathrm{B}$.

\subsection{Dyon string condensation in more detail}

The actual construction of the nontrivial gapped bulk is as follows. Again each layer is ordinary electrodynamics with bosonic charges. We will call $\epsilon_{i} \equiv e_{i} m_{i}$ the dyon in each layer, which is a fermion. The object $b_{i} \equiv \epsilon_{i}^{\dagger} m_{i+1} \epsilon_{i+2}$ is a boson (two fermions plus one boson, and no net electric charge to produce extra statistics, equals a boson).

The objects $b_{i}(i=1 . . N-2)$, for all $i$, are mutually local (i.e. their charge vectors satisfy $q_{i} e_{j}-q_{j} e_{i}=0, \forall i, j=1 . . N-2$ ) under the total $U(1)$ (in particular, they all have $q_{e}^{\text {Total }}=0, q_{m}^{\text {Total }}=1$ ). This means that it is possible to couple the layers so that these objects condense [71-73]. 
Explicitly, we can cause them to condense by adding the completely local gauge invariant hamiltonian $\Delta H=V \sum_{x} \sum_{i}\left(\left|b_{i}(x)\right|^{2}-v^{2}\right)^{2}$. The phase of the condensate $b_{i}(x)=v e^{\mathbf{i} a_{i, i+2}}$ is again a link variable along the extra dimension; unlike the simple construction of $\S 4.1$, the duality frame in which this object is the vector potential rotates as we increase $i$.

Condensing $b_{i}$ (obliquely) confines the gauge fields in the layers $a_{i+1}, i+1=2 \ldots N-1$. Objects which are not mutually local with $b_{i}$ are confined. What's left? We are condensing $N-2$ objects in a theory with gauge group $\mathrm{U}(1)^{N}$, so two gauge fields remain massless. The charged objects which are mutually local with the condensate and therefore not confined [71-73] are (just as in the $2 \mathrm{~d} \mathbb{Z}_{2}$ case [6]):

- At the top layer : $\epsilon_{1}, m_{1} \epsilon_{2}^{\dagger}$ and their boundstate $\epsilon_{1} m_{1} \epsilon_{2}^{\dagger}$ (and powers and products of these) and

- At the bottom layer: $\epsilon_{N}, m_{N-1} \epsilon_{N}^{\dagger}, \epsilon_{N} m_{N-1} \epsilon_{N}^{\dagger}$ etc.

At the top layer, the objects $\epsilon_{1}, m_{1} \epsilon_{2}^{\dagger}$ are both fermions, and have charge $\left(q_{e}, q_{m}\right)=$ $(1,1)$ and $(-1,0)$ respectively. The boundstate has charge $(0,1)$ and is therefore also a fermion, by the standard argument reviewed above, because there is still a Maxwell field at the top layer.

To see the full effect of condensing $b_{i}$, consider the blue box in the figure at right. Although $\epsilon_{i}^{\dagger} m_{i+1}$ is mutually local with $m_{i} \epsilon_{i+1}$, the constituents are not. This has the consequence that condensing $b_{i}$ binds the monopole strings of $a^{e / o}$ to electric flux lines of $a^{o / e}$ ! This is precisely the effect of the additional term

$$
\Delta S=\int \frac{1}{2 \pi} C^{e} \wedge d C^{o} \equiv \int \frac{1}{2 \pi} B \wedge d C
$$

in the low-energy description.

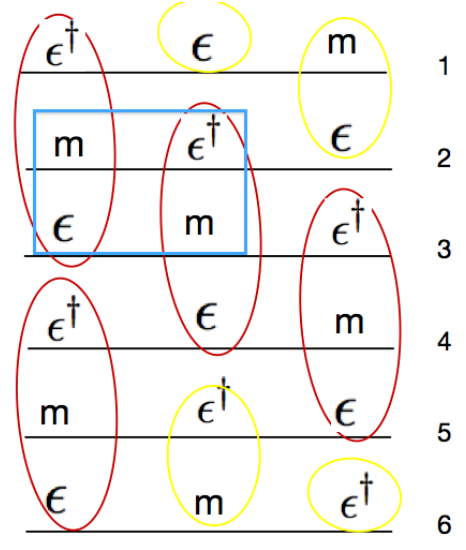

\subsection{Alternative description of layer construction}

Here we make contact between the coupled layer construction of the previous subsection and the general description (in the section introduction) in terms of coupled layers of $\mathcal{E}$ and $\mathcal{E}^{-1}$ which guarantees the correct edge states.

Again let $\mathcal{E}$ denote a single copy of all-fermion electrodynamics. First we note that the all-fermion electrodynamics is its own inverse: $\mathcal{E}=\mathcal{E}^{-1}$ in the sense that two copies of all-fermion electrodynamics can be regularized in $3+1$ dimensions. More specifically, $\mathcal{E} \times \mathcal{E}$ is deformable (by adding local, gauge-invariant interactions) to ordinary bosonic 
$\mathrm{U}(1)$ gauge theory. To see this ${ }^{10}$, let $e$ and $\tilde{e}$ denote the electrons in $\mathcal{E}$ and $\mathcal{E}^{-1}$. Define $b=e \tilde{e}^{\dagger}$, which is a boson. If we condense this boson, we higgs $U(1)_{\mathcal{E}} \times U(1)_{\mathcal{E}^{-1}}$ to the diagonal $\mathrm{U}(1)$ subgroup. The object $e$ is a fermion charged under this gauge group; it is related to $\tilde{e}$ by taking charges from the condensate. We should think of this object as the dyon of ordinary BBF electrodynamics, because all of the other excitations which are mutually local with the condensate are bosons:

- $m, \tilde{m}, \epsilon=e m$ and $\tilde{\epsilon}=\tilde{e} \tilde{m}$ are non-local with respect to the condensate, so they are confined.

- $M \equiv m \tilde{m}^{\dagger}$ is a boson which differs from $e$ by one unit of electric charge, and so we should think of it as the monopole. It is related by taking stuff from the condensate to $\epsilon \tilde{\epsilon}$.

- Adding $e$ to $M$ we get another boson (since we are combining two mutually non-local fermions) $\epsilon \tilde{m}^{\dagger}$; apparently we should regard this as the elementary electrically charged boson.

We conclude that $\mathcal{E} \times \mathcal{E}^{-1}$ is separated by simple Higgs transition from the phase $U(1)_{F B B}$, with a propagating photon (if it's in the deconfined phase), and therefore has a $D=3+1$ regulator.

It is important to note that the remaining electrodynamics still has charged matter which may be condensed to higgs or confine the photon; the choice of whom to condense means that various bulk models are possible.

So, while a single copy of all-fermion electrodynamics cannot be regulated in $3+1$ dimensions, a pair of copies can be so regulated since $U(1)_{F B B}$ can be so regulated and $\mathcal{E} \times \mathcal{E}^{-1} \sim U(1)_{F B B}$. The layer construction in $\S 4.2$, when applied a slab of finite thickness, provides just such a regulator. As long as the thickness of the slab is not taken to infinity, the two copies of all-fermion electrodynamics can be regarded as living in $3+1$ dimensions.

Further insight into the layer construction is obtained by viewing the construction in terms of a stack of such slabs, where each slab, denoted $\left(\mathcal{E}^{-1}\right)$, hosts two copies of all-fermion electrodynamics, one on the bottom surface and one on the top surface. The stack of slabs is denoted $\left(\mathcal{E}_{1} \mathcal{E}_{1}^{-1}\right) \ldots\left(\mathcal{E}_{n} \mathcal{E}_{n}^{-1}\right)$ where $i=1, \ldots, n$ indicates the extra spatial dimension. Pairing up the all-fermion states within each slab produces the trivial bulk state in $4+1$ dimensions. Pairing $\mathcal{E}_{i}^{-1}$ with $\mathcal{E}_{i+1}$ across neighboring slabs realizes the bulk non-trivial state. This way of thinking about the layer construction realizes the motivating idea given in the section introduction.

\footnotetext{
${ }^{10} \mathrm{An}$ essentially identical argument shows that the all-fermion toric code is its own inverse.
} 
To be a little more explicit, condensing only $b_{i}=\left(e_{i \text {,top }} \tilde{e}_{i \text {,bottom }}^{\dagger}\right)$ produces layers of ordinary $F B B$ electrodynamics, by the preceding argument. This returns us to the starting point of the layer construction of the previous section. The slabs of $F B B$ electrodynamics can then be confined to produce a trivial bulk state.

To produce the non-trivial bulk state, the gluing may be performed by repairing the missing condensates at the top and bottom of Fig. 2. In particular, think of each pair $\mathcal{E}_{i} \mathcal{E}_{i}^{-1}$ as a copy of Fig. 2. At the top we have fermionic charges $\epsilon_{1}$ and $m_{1} \epsilon_{2}$; at the bottom we have fermionic charges $\epsilon_{N-1}^{\dagger} m_{N}$ and $\epsilon_{N}^{\dagger}$. If we glue the bottom to the top by condensing

$$
b_{N-1} \equiv\left(\epsilon_{N-1}^{\dagger} m_{N}\right) \epsilon_{1}
$$

and

$$
b_{N} \equiv \epsilon_{N}^{\dagger}\left(m_{1} \epsilon_{2}\right)
$$

then we get the $\mathrm{BdC}$ theory rolled up on a circle, i.e. the coupled layer construction has translation invariance $i \rightarrow i+1$. And in particular, there is no photon in the bulk.

\subsection{Extension to $D=3+1$ and derivation of $B F$ theory}

The logic by which we inferred the presence of the $B d C$ coupling from the coupled layers construction can be applied to the original construction [6] of the $D=3+1$ boson SPT state with time-reversal symmetry. The string of magnetic excitations is a vortex line; the mutual nonlocality of the constituents of the condensed boson glues this vortex line to the electric flux lines of the other gauge field. The result is that the

bulk model contains a term of the form $\frac{1}{2 \pi} B \wedge F$. That the bulk theory admits such an effective description is well-known [37]. An implication of this derivation which has not been appreciated to our knowledge is that the all-fermion toric code - when realized on the surface of a bosonic SPT - suffers a fermion-number anomaly, as we discuss in the next section.

\section{Fermion number anomaly inflow}

We will now interpret the obstruction studied here in terms of global anomaly inflow. The only symmetry involved in this system is fermion parity. We emphasize that in the bulk there are no fermions; however, the Jackiw-Rebbi effect demonstrates clearly that gauge fields are capable of carrying this quantum number.

In the following we show that the fermion number conservation on the surface of the $4+1 d$ short-range-entangled state constructed in the previous section is violated by high-energy processes. 
There is a precedent for such violation of fermion number by quantum gauge theory. The Witten SU(2) anomaly [74] can be regarded as an anomaly for fermion number: in a Witten-anomalous gauge theory, instanton events create an odd number of fermions and hence violate fermion parity conservation; this is not something we know how to describe with a local field theory.

In the prehistory of SPT physics, a subset of the authors [75] studied a system where the Witten anomaly played a crucial role in preserving the integrity of the classification of statistics of $3+1 \mathrm{~d}$ particles. In particular, the Witten anomaly was argued to forbid a gauge theory whose monopoles carry a single majorana zero mode (which monopoles, if they could be deconfined, would enjoy non-Abelian statistics). That paper also described a $4+1 \mathrm{~d}$ dimensional model whose edge realized such a gauge theory, and therefore could be regarded as exhibiting 'Witten anomaly inflow'.

Fermion number anomaly. The all-fermion electrodynamics, as it arises on a surface of the coupled-layer construction, exhibits crucial differences from an intrinsically 3+1-dimensional system with a bosonic regulator. First of all, note that the slab geometry constructed in $\S 4$ harbors gauge-invariant states with a single fermionic particle at the top layer ${ }^{11}$. Since all femionic excitations carry some gauge charge (either electric or magnetic) - as they must in a system with a bosonic regulator - there is no state in a putative $3+1$ dimensional realization of this form.

Further, the coupled layer construction of $\S 4$ directly shows that fermion number can be transported across the extra dimension, as follows. Consider a state with an excitation of $\epsilon_{1}$, the dyon at the top layer. This excitation can for free absorb bosons from the condensate, which include objects of the form $b_{1}=\epsilon_{1}^{\dagger} m_{2} \epsilon_{3}$. Combining these two objects we get something with the quantum numbers of $m_{2} \epsilon_{3}$. This looks a bit like a bulk fermion excitation, but this object is confined (since it is not mutually local

${ }^{11}$ Here we are assuming that the $3 \mathrm{~d}$ geometry is noncompact, so that the flux has somewhere to go. If the $3 \mathrm{~d}$ spatial sections are compact, we cannot have a single string stretching from one end of the slab to the other because of the bulk Gauss law:

$$
0=\frac{\delta S}{\delta B}=\star j+d C+d \star d B \frac{1}{g^{2}}
$$

which is a 3 -form. If we integrate this over a $3 \mathrm{~d}$ region $\Upsilon$ at fixed time and codimension 1 in space, we get

$$
0=(\text { number of strings penetrating the region, counted with orientation })+\int_{\partial \Upsilon}\left(C+\star d B / g^{2}\right) .
$$

The last term is the usual Gauss' law term for a 2-form potential, but the important thing is that the dependence on the fields on the RHS of (11) is a total derivative. So if there is no boundary of $\Upsilon$ such as if the whole space is $T^{3} \times(0,1)$ and we choose $\Upsilon$ to be the $T^{3}$ at some fixed position along the interval - then the net number of strings must be zero. 
with $b_{2}$, which is condensed). Also condensed is $b_{3}=\epsilon_{3}^{\dagger} m_{4} \epsilon_{5}$; adding one of these in, we get $m_{2} m_{4} \epsilon_{5}$. The bottom layer (for argument, we take $N=6$ layers, as in the figure

above) supports a deconfined fermion excitation $\epsilon_{5}^{\dagger} m_{6}=f_{\text {bottom. }}$. The condensate plus top-layer excitation $f_{\text {top }}=\epsilon_{1}$ is related to this by

$$
f_{\text {top }} b_{1} b_{3}=m_{2} m_{4} m_{6} f_{\text {bottom }}^{\dagger}
$$

With arbitrary (even) $N$, we have:

$$
f_{\text {top }} b_{1} b_{3} \ldots b_{N / 2}=m_{2} m_{4} \ldots m_{N} f_{\text {bottom }}^{\dagger}
$$

This equation is understood to be true modulo the creation of neutral excitations (which are all bosonic, by assumption).

This strongly suggests that a monopole string $\left(m_{2} m_{4} m_{6} \ldots\right)$ (bosonic, but confined) allows fermions to tunnel from the top layer to the bottom layer. A quantitative statement to this effect is that there is a nonzero amplitude in the groundstate $|\mathrm{gs}\rangle$ for a pair of fermions to be created at top and bottom, connected by a monopole string:

$$
\left\langle\mathrm{gs}\left|f_{\mathrm{bottom}} m_{2} m_{4} \ldots m_{N} f_{\mathrm{top}}^{\dagger}\right| \mathrm{gs}\right\rangle=\left\langle\mathrm{gs}\left|f_{\mathrm{top}} f_{\mathrm{top}}^{\dagger} b_{1} b_{3} \ldots b_{N / 2}\right| \mathrm{gs}\right\rangle=v^{n}\left\langle\mathrm{gs}\left|f_{\mathrm{top}} f_{\mathrm{top}}^{\dagger}\right| \mathrm{gs}\right\rangle \neq 0
$$

$v^{n} \sim e^{-L}$ decays exponentially in the thickness of the slab, but this implies a finite tunneling amplitude. (Here $n(N) \equiv \frac{N-2}{2}$.)

Since all fermions are charged either electrically or magnetically (it is ambiguous which should be interpreted as the electron and which as the magnetic monopole), the fermion number anomaly also implies a discrete gauge anomaly. That is, rotating the phase of every fermion by $\pi$ is part of the $\mathrm{U}(1)$ gauge group (though not only the electric group in any one duality frame). This is similar to Goldstone's understanding of the Witten anomaly [76] (as cited in [77-79]).

Putting two copies of the system together removes the anomaly. From the point of view above, it is because the monopole strings will reconnect so that they only attach fermions at the same surface. A similar mechanism of reconnection was described in $[75]$.

\section{Consequences for all-fermion toric code}

So far we've discussed bosonic SPTs in $D=4+1$ with no symmetry, and have briefly mentioned bosonic SPTs in $D=3+1$ with time-reversal $(\mathcal{T})$ symmetry. In both cases, there is a symmetry-preserving termination which is a gauge theory where all the matter is fermionic. There are many illuminating connections between these two 
problems. To understand them, we must now discuss the $D=3+1 \mathcal{T}$ invariant SPT $[6,15,17,37,38]$ in more detail.

Briefly, the bulk $3+1$ dimensional state is a quantum phase of bosons protected by time reversal symmetry. The bulk theory has a surface termination consisting of $2+1$ dimensional $\mathbb{Z}_{2}$ gauge theory in which the charge, the vortex, and chargevortex composite are all fermions. As in the case of all-fermion electrodynamics, the statistics of the charge-vortex composite actually follows from those of the charge and the vortex provided there are no gauge-invariant fermions in the spectrum. What does time reversal have to do with such a $2+1$ dimensional state? Naively, the answer is not much: all topological data, e.g., fusion rules, quantum dimensions, braiding phases, etc. are real numbers, so time reversal invariance doesn't seem to provide a constraint on the topological data.

However, there is one piece of topological data which is sensitive to $\mathcal{T}$ and that is the chiral central charge, $c_{-}$. Furthermore, in a microscopic bosonic model, the value of $c_{-}$is constrained by the topological data. If we have anyon types labeled by $a$ with quantum dimensions $d_{a}$ and topological spins $s_{a}$, then the chiral central charge is determined, $\bmod 8$, by [80-82]

$$
\frac{\sum_{a} d_{a}^{2} e^{2 \pi i s_{a}}}{\sqrt{\sum_{a} d_{a}^{2}}}=e^{2 \pi i c_{-} / 8} .
$$

In a model of abelian anyons, all $d_{a}=1$ and the total quantum dimension, $\mathcal{D}=$ $\sqrt{\sum_{a} d_{a}^{2}}$, is simply the square root of the number of anyon types (including the identity). The fact that the central charge is only determine mod 8 is not an accident [82]. The $\mathrm{E}_{8}$ state of bosons has no anyonic excitations but has chiral central charge $c_{-}=8$, hence we may add layers of the $\mathrm{E}_{8}$ to any anyon model without changing the anyon content but shifting the chiral central charge by 8 .

For the familiar $\mathbb{Z}_{2}$ gauge theory in which charges and vortices are bosons, we have $a \in\{1, e, m, e m\}, d_{a}=1, s_{1}=s_{e}=s_{m}=0$, and $s_{e m}=1 / 2$. Hence (12) gives

$$
e^{2 \pi i c_{-} / 8}=\frac{3+(-1)}{2}=1
$$

hence $c_{-}=0 \bmod 8$. In other words, the minimal $\mathbb{Z}_{2}$ gauge theory has no chiral edge states. However, if we consider the all-fermion gauge theory, then we find

$$
e^{2 \pi i c_{-} / 8}=\frac{1+3(-1)}{2}=-1
$$

hence $c_{-}=4 \bmod 8$. Thus the all-fermion gauge theory must have chiral edge states and hence must indeed break $\mathcal{T}$. The reason why this state can be realized in a $\mathcal{T}$ invariant manner at the surface of a $\mathcal{T}$-invariant $3+1$ bulk state is that in this case 
it is impossible to create an edge for the gauge theory at which the chiral edge states can be exposed.

Now we turn to connections between the system just discussed and the all-fermion electrodynamics in $D=3+1$. First, suppose all-fermion electrodynamics did have a time reversal symmetric bosonic regulator. Then so does the all-fermion toric code. The argument is as follows. Condense pairs of charges in $3+1 \mathrm{~d}$ (thereby higgsing the gauge group to $\mathbb{Z}_{2}$ ), and place the system on $\mathbb{R}^{2} \times S^{1}$, where the radius of the $S^{1}$ is $L$. The $\mathbb{Z}_{2}$ topological order implies that states with different $\mathbb{Z}_{2}$ flux through the circle are split only by an amount of order $E_{\text {flux }} \sim e^{-L|\log t| / \xi}$ where $t$ is a hopping amplitude for $\mathbb{Z}_{2}$ charged quasiparticles, and $\xi$ is the bulk correlation length. The regime of interest has $L \gg \xi$ (so that our field theory analysis is valid) and $E_{\text {flux }} \gg m_{e}, m_{m}$, where $m_{e}$ and $m_{m}$ are the rest energies of the electric and magnetic quasiparticle excitations. The result is then the all-fermionic toric code with, by assumption, a time-reversal symmetric bosonic regulator. Assuming that no such regulator exists for the all-fermion toric code, no such regulator can exist for all-fermion electrodynamics. (And as [1] point out, the case with time reversal symmetry is actually the crucial case, in the sense that the SPTness of the state persists even upon breaking time reversal.)

Second, all-fermion electrodynamics does have a time reversal symmetric fermionic regulator. Indeed, it is equivalent to BBF electrodynamics by binding the neutral fermion to the electron. (In this case there are particles of both statistics in each charge sector; for purposes of discussion, we label a model by the statistics of the lightest particle in each sector.) Again condense charges and compactify on a circle. This produces a time reversal symmetric fermionic regulator for the all-fermion toric code. And again, we can convert FFF toric code to BBF toric code in the process.

It is instructive to ask what happens to the chiral central charge formula (12). The answer is that the formula only applies when the regulator is bosonic. This is crucial because the mod 8 property of the formula relied on the $\mathrm{E}_{8}$ phase being the simplest phase with chiral edge states and no anyonic excitations. Once we add microscopic fermions, there are simpler chiral states. The simplest is the $p+i p$ state of fermions with $c_{-}=1 / 2$. Hence while the minimal chiral central charge, $c_{-}=4$, of the allfermion gauge theory could not be cancelled with only bosonic short-range entangled states (which can only shift $c_{-}$by 8 ), the minimal central charge of the all-fermion gauge theory can be cancelled by fermionic short-range entangled states (which can shift $c_{-}$by $\left.1 / 2\right)$.

In both cases adding microscopic fermions saves everything, in the sense that all spectra of excitations are adiabatically connected. 
Fermion number anomaly. Since the structure of our coupled-layer construction is so similar to that of the $D=3+1$ beyond-cohomology boson SPT in [6], the same logic applies to that model (removing daggers where necessary since charges are binary). That is, in a slab geometry, a state with a fermion on the top surface can tunnel to a state with a fermion on the bottom surface, because the quasiparticle sectors are related by bosonic operators (some of which are condensed):

$$
f_{\text {top }} b_{1} b_{3} \ldots b_{N / 2}=m_{2} m_{4} \ldots m_{N} f_{\text {bottom }}
$$

We therefore expect that this bosonic state can transport fermion number between edges.

In this case, the bulk state is protected by time-reversal invariance. Breaking time reversal only at the surface produces a state which is still not edgeable. We give two examples of time-reversal broken surface states momentarily. It will help to see the connection between the fermion number anomaly and the preservation of $\mathcal{T}$ to ask: What happens to the edge if we adiabatically continue the bulk through a $\mathcal{T}$-breaking path to a product state? It is not necessary to have a surface phase transition: Without $\mathcal{T}$, one way to deform the bulk (on a torus, say) to a product state is to open up an array of gapped trivial surfaces (possible because $\mathcal{T}$ is broken) and then expand the intervening vacuum regions to consume the system, following [36]. On a system with boundary, this can be done everywhere except at topologically ordered boundaries which are independently stable. On a slab of finite but large thickness, therefore, in the absence of $\mathcal{T}$, one can disconnect the top from the bottom by cutting open a middle (trivial, gapped) surface, hence ending the fermion tunneling without destroying the surface topological order.

A model with the same spectrum of quasiparticles and braiding statistics can be realized intrinsically in $D=2+1$. For example, it can be obtained from the Kitaev honeycomb model with $\nu=8$ (see Table 2 of [82]). That model does not preserve time reversal symmetry: the violations of time-reversal symmetry occur at boundaries, where there is a chiral edge spectrum (with $c_{L}-c_{R}=4$ ). The model at the surface of the boson SPT cannot be put on a space with boundary (since the boundary of a boundary is empty) and is time-reversal invariant. The price for this extra symmetry is that the fermion number is not conserved!

To connect the various phenomena, it is useful to explicitly realize various $\mathcal{T}$ broken surface states starting from the $\mathcal{T}$ invariant all-fermion surface toric code. The basic observation follows from the previous paragraph: given $\mathbb{Z}_{2}$ charged fermionic matter we may shift the vortex from bosonic to fermionic and vice versa by adding $\nu= \pm 8$ copies of a $p+i p$ state for the charged fermions. Normally in $2+1$ dimensions the timereversal point has an absolute chiral central charge $c_{-}=0$ and a bosonic vortex. We 
can obtain a fermionic vortex and $c_{-}=4$ by adding $\nu=8$ copies of charged fermions in $p+i p$ states. However, on the surface of the $\mathcal{T}$ invariant bosonic SPT, there is a shift in the spectrum so that the $\mathcal{T}$ invariant point has a fermionic vortex. Then we may construct a pair of $\mathcal{T}$ broken surface states which are still topologically ordered by adding $\nu= \pm 8$ copies charged fermions in $p+i p$ states. The system now explicitly breaks time reversal and has a bosonic vortex.

Given a bosonic vortex, we may condense the vortex to destroy the surface topological order. At a domain wall between the two distinct ways to break $\mathcal{T}$ to obtain a bosonic vortex we have $\nu=16$ Majorana edge modes before condensing the vortex. After condensing the vortex we obtain the edge of $E_{8}$ state of bosons [30]. Thus we obtain the same edge physics as the $\mathrm{E}_{8}$ BF theory discussed in [37]. This analysis provides another route to connect the layer construction to a topological bulk theory via the non-trivial surface, in this case in $3+1$ dimensions. When the surface preserves $\mathcal{T}$ we may interpret the bulk $F F$ term in $3+1$ dimensions as providing a $\mathcal{T}$ invariant regulator for the surface all-fermion toric code.

Again the presence of neutral bulk fermions renders everything trivial. In the presence of microscopic neutral fermions, the bosonic SPT can be deformed into 16 copies of the free fermion topological superconductor, and this in turn is equivalent to nothing $[35,83]$. So adding fermions explicitly makes the bulk trivial (in addition to the edge). This picture nicely complements the edge analysis above where we argued that adding fermions effectively changes the minimal chiral central charge one can have without topological order (from $c_{-}=8$ to $c_{-}=1 / 2$ ).

Reality of this phenomenon. We have to ask: Are there real physical systems made just of bosons, with a gap, which can transport fermion number? The $D=3+1$ boson SPT protected by time-reversal should do so. This makes it even more interesting to try to realize this state in the world.

Finally, we note the following consequence of our claim, given that elementary gauge-neutral fermions have not been observed in nature ${ }^{12}$. Were we to discover a fermionic magnetic monopole in our world, it would imply either ${ }^{13}$ :

1. There are microscopic, gauge-neutral fermions. The opposite is conjectured to be true in e.g. Ref. [53].

2. We live on the boundary of some higher dimensional space. Boundary theories of $4+1 \mathrm{D}$ SPT phases have been suggested in attempts to understand the matter content (and flavor structure) of the standard model [84-87].

\footnotetext{
${ }^{12}$ Here we mean 'neutral under gauge groups which are unbroken at low energies'; absent discrete gauge symmetries, a right-handed neutrino would falsify this claim.

${ }^{13}$ We must note some uncertainty involving the role of gravity.
} 
Acknowledgements. We thank C. Chamon, D. Das, B. Dolan, J. Kaplan, and T. Senthil for useful discussions and comments. This work was supported in part by funds provided by the U.S. Department of Energy (D.O.E.) under cooperative research agreement DE-FG0205ER41360, in part by the Alfred P. Sloan Foundation. BGS and JAM acknowledge the hospitality of the Perimeter Institute for Theoretical Physics during the workshop "Low Energy Challenges for High Energy Physicists". Research at Perimeter Institute is supported by the Government of Canada through Industry Canada and by the Province of Ontario through the Ministry of Economic Development \& Innovation.

\section{A Lattice bosons for duality-symmetric surface QED}

This is a model of bosons. The two-form gauge theory studied in this paper is a model of bosons. Low-energy evidence for this statement is the fact that we did not have to choose a spin structure to put it on an arbitrary 4-manifold. This is in contradistinction to $\mathrm{U}(1)_{k=1}$ CS theory in $D=2+1$. We note in passing that on a manifold that admits spinors, the intersection form is even $(\mathcal{I}(v, v) \in 2 \mathbb{Z})$ [88]. (This means that to describe an effective field theory for a fermionic SPT state, we should consider the level $k \in \mathbb{Z} / 2$.)

High-energy (i.e. condensed-matter) evidence for the claim that this is a model of bosons is the following conjecture for a lattice model of bosons which produces this EFT. The Hilbert space is as follows and is similar to lattice boson constructions of electrodynamics in other dimensions [89-92].

- Put rotors $e^{\mathrm{i} b_{p}}$ on the plaquettes $p$ of a $4 \mathrm{~d}$ spatial lattice. (Actually, the model is defined for any $4 \mathrm{~d}$ simplicial complex. Translation invariance will not play a significant role.) These act as

$$
e^{\mathbf{i} b_{p}}\left|n_{p}\right\rangle=\left|n_{p}+1\right\rangle
$$

on states with definite excitation number $n_{p}$; we will interpret $n_{p}$ as a number of (oriented) 'sheets' covering the plaquette.

- Put charge- $k$ bosons $\Phi_{\ell}=\Phi_{-\ell}^{\dagger}$ on the links $\ell$. These satisfy $\left[\Phi_{\ell}, \Phi_{\ell}^{\dagger}\right]=1$. We will say that $\Phi_{\ell}^{\dagger}$ creates a string segment, and $\Phi_{\ell}^{\dagger} \Phi_{\ell}$ is the number of (oriented) strings covering the link.

The Hamiltonian is

$$
\mathbf{H}=-\underbrace{\sum_{\text {links, } \ell \in \Delta_{1}}\left(\sum_{p \in s(\ell)} n_{p}-k \Phi_{\ell}^{\dagger} \Phi_{\ell}\right)^{2}}_{\mathbf{H}_{1}, \begin{array}{c}
\text { gauss law. happy when sheets close, } \\
\text { or end on strings }
\end{array}}-\underbrace{\sum_{\text {volumes, } v \in \Delta_{3}} \prod_{p \in \partial v} e^{\mathbf{i} b_{p}}+h . c .}_{\mathbf{H}_{3} \sim B^{2}, \text { makes sheets hop. }}
$$




$$
-\underbrace{\Gamma \sum_{p \in \Delta_{2}} n_{p}^{2}}_{\mathbf{H}_{2} \sim E^{2} \text {. discourages sheets. }}-\underbrace{t}_{\mathbf{H}_{\text {strings }}, t \sum_{p \in \Delta_{2}} e^{\mathrm{i} k b_{p}} \prod_{\ell \in \partial p} \Phi_{\ell}^{\dagger}+h . c .}+V\left(|\Phi|^{2}\right)
$$

The subscripts indicate the dimension of the simplices to which the terms are associated. When $\Gamma=0, V=0$, these terms all commute. The groundstate for $t>0$ is described by a soup of oriented closed $2 \mathrm{~d}$ sheets, groups of $k$ can end on strings.

Now take $V\left(|\Phi|^{2}\right)=\left(|\Phi|^{2}-v^{2}\right)$. This causes to condense $\Phi_{\ell}=v e^{\mathbf{i} \varphi \ell}$, which leads to a 2-form higgs mechanism:

$$
\mathbf{H}_{\text {strings }}=-\sum_{p} t v^{4} \cos \left(k b_{p}-\sum_{\ell \in \partial p} \varphi_{\ell}\right)
$$

On the low-energy manifold of this Hamiltonian, we have

$$
\left(e^{\mathrm{i} b_{p}}\right)^{k}=11, \quad\left|n_{p}\right\rangle \simeq\left|n_{p}+k\right\rangle .
$$

This leaves behind $k$ species of (unoriented) sheets.

The groundstates are then described by equal-superposition sheet soup. If the intersection form on the spatial 4-manifold which is triangulated by the simplicial complex has $\mathcal{I}=\mathbb{1}$, there are $k^{b_{2}}$ groundstate sectors. These groundstates represent the algebra of 'tube operators': for any closed union of 2 -simplices $\omega$

$$
\begin{gathered}
\mathcal{F}_{\omega} \equiv \prod_{p \in \omega} e^{\mathrm{i} b_{p}} \quad \mathcal{T}_{\omega} \equiv \prod_{V \in \Delta_{3}} \prod_{p \in \partial V \cap \omega} n_{p} \\
\mathcal{F}_{\omega} \mathcal{T}_{\omega^{\prime}}=e^{2 \pi \mathbf{i} \mathcal{I}_{\omega \omega^{\prime}}} \mathcal{T}_{\omega}^{\prime} \mathcal{F}_{\omega}
\end{gathered}
$$

Continuum limit. The higgs mechanism described above leads to $U(1) \stackrel{\text { higgs }}{\rightarrow} \mathbb{Z}_{k}$ 2-form gauge theory:

$$
\begin{aligned}
L= & \frac{t v^{4}}{2}\left(\mathrm{~d} \varphi_{1}+k B_{2}\right) \wedge \star\left(\mathrm{d} \varphi_{1}+k B_{2}\right)+\frac{1}{g^{2}} \mathrm{~d} B_{2} \wedge \star \mathrm{d} B_{2} \\
& \simeq \frac{k}{2 \pi} B \wedge \mathrm{d} C+\frac{1}{8 \pi t v^{4}} d C \wedge \star \mathrm{d} C+\frac{1}{g^{2}} \mathrm{~d} B \wedge \star \mathrm{d} B
\end{aligned}
$$

with $\mathrm{d} C \simeq 2 \pi t \star(\mathrm{d} \varphi+k B)$. This equivalence is described in [51,93]. 


\section{B More details on monopole strings and vortex sheets in $5 \mathrm{~d}$ abelian gauge theory}

Consider a $5 \mathrm{~d} \mathrm{U}(1)$ 1-form gauge field $a$, with field strength $f=d a$. A magnetic

excitation with respect to this gauge field has $\oint_{\Sigma_{2}} f=2 \pi g$, where $\Sigma_{2}$ is a closed 2surface surrounding the object. Such an object is therefore codimension three, and is a string in $4+1$ dimensions. The quantity which is localized on the monopole strings is therefore a three-form:

$$
\star j_{m}=\delta^{3} \text { (monopole strings) }=\star d f \equiv d C
$$

where $C$ is a two-form.

Suppose we higgs the $U(1)$ gauge field by condensing a charged order parameter field $b \sim v e^{i \phi}$. This adds

$$
\delta H=m^{2}(a+d \phi)^{2},
$$

so that $a$ eats the phase $\phi$, and $m \sim t v$. Topological defects in $\phi$, i.e. zeros of $b$ around which $\phi$ winds by $2 \pi$, occur at codimension two (since $b$ is a complex function) and in $5 \mathrm{~d}$ are therefore 2+1-dimensional vortex sheets.

These vortex sheets can end on the monopole strings. This is the same fact as the fact that vortex strings can end on magnetic monopoles in $3+1$ dimensions. In the higgs phase of a $3+1$ dimensional abelian gauge theory, the vortex string provides a means to collimate the magnetic flux coming out of the monopole. The result is the confinement of the magnetic charges; this is a manifestation of the Meissner effect. It is the same in $D=4+1$, except now it is magnetically charged strings which are connected by vortex sheets. In the higgs phase, it is energetically favorable for the monopole strings to be connected by such vortex sheets.

The final ingredient in the coupled-layer construction is the fact that the condensate is not purely electric with respect to any individual layer.

\section{References}

[1] C. Wang, A. C. Potter, and T. Senthil, "Classification of Interacting Electronic Topological Insulators in Three Dimensions," Science 343 (Feb., 2014) 629-631, 1306.3238.

[2] X.-G. Wen, "Topological order: from long-range entangled quantum matter to an unification of light and electrons," 1210.1281. 
[3] B. I. Halperin, "Quantized Hall conductance, current-carrying edge states, and the existence of extended states in a two-dimensional disordered potential," Phys. Rev. B 25 (Feb, 1982) 2185-2190, http://link.aps.org/doi/10.1103/PhysRevB.25.2185.

[4] A. M. Turner and A. Vishwanath, "Beyond Band Insulators: Topology of Semi-metals and Interacting Phases," 1301.0330.

[5] T. Senthil, "Symmetry Protected Topological phases of Quantum Matter," 1405.4015.

[6] C. Wang and T. Senthil, "Boson topological insulators: A window into highly entangled quantum phases," Phys. Rev. B 87 (Jun, 2013) 235122, http://link.aps.org/doi/10.1103/PhysRevB.87.235122.

[7] S. M. Kravec and J. McGreevy, "Gauge theory generalization of the fermion doubling theorem," Phys. Rev. Lett. 111 (Oct, 2013) 161603, 1306.3992, http://link.aps.org/doi/10.1103/PhysRevLett.111.161603.

[8] G. 't Hooft, "Naturalness, chiral symmetry, and spontaneous chiral symmetry breaking," NATO Adv.Study Inst.Ser.B Phys. 59 (1980) 135.

[9] J. Callan, Curtis G. and J. A. Harvey, "Anomalies and Fermion Zero Modes on Strings and Domain Walls," Nucl.Phys. B250 (1985) 427.

[10] J. Wang, L. H. Santos, and X.-G. Wen, "Bosonic Anomaly, Induced Fractional Quantum Number and Degenerate Zero Modes - the anomalous edge physics of Symmetry Protected Topological States," 1403.5256.

[11] A. Kapustin, "Bosonic Topological Insulators and Paramagnets: a view from cobordisms," 1404.6659.

[12] A. Kapustin and R. Thorngren, "Anomalies of discrete symmetries in various dimensions and group cohomology," 1404.3230.

[13] A. Kapustin, "Symmetry Protected Topological Phases, Anomalies, and Cobordisms: Beyond Group Cohomology," 1403.1467.

[14] A. Kapustin and R. Thorngren, "Anomalies of discrete symmetries in three dimensions and group cohomology," 1403.0617.

[15] F. Burnell, X. Chen, L. Fidkowski, and A. Vishwanath, "Exactly Soluble Model of a 3D Symmetry Protected Topological Phase of Bosons with Surface Topological Order," 1302.7072. 
[16] C. Wang, A. C. Potter, and T. Senthil, "Gapped symmetry preserving surface state for the electron topological insulator," Phys. Rev. B $8 \mathbf{8}$ (Sep, 2013) 115137, http://link.aps.org/doi/10.1103/PhysRevB.88.115137.

[17] M. A. Metlitski, C. Kane, and M. P. A. Fisher, "Bosonic topological insulator in three dimensions and the statistical Witten effect," 1302.6535.

[18] P. Bonderson, C. Nayak, and X.-L. Qi, "A time-reversal invariant topological phase at the surface of a 3D topological insulator," J.Stat.Mech. 2013 (2013) P09016.

[19] L. Fidkowski, X. Chen, and A. Vishwanath, "Non-Abelian Topological Order on the Surface of a 3D Topological Superconductor from an Exactly Solved Model," Physical Review X 3 (Oct., 2013) 041016, 1305.5851.

[20] X. Chen, F. J. Burnell, A. Vishwanath, and L. Fidkowski, "Anomalous Symmetry Fractionalization and Surface Topological Order," ArXiv e-prints (Mar., 2014) 1403.6491.

[21] M. A. Metlitski, C. L. Kane, and M. P. A. Fisher, "A symmetry-respecting topologically-ordered surface phase of $3 \mathrm{~d}$ electron topological insulators," ArXiv e-prints (June, 2013) 1306.3286.

[22] A. Amoretti, A. Braggio, G. Caruso, N. Maggiore, and N. Magnoli, "Holography in flat spacetime: 4D theories and electromagnetic duality on the border," JHEP 1404 (2014) 142, 1401.7101.

[23] J. H. Schwarz and A. Sen, "Duality symmetric actions," Nucl.Phys. B411 (1994) 35-63, hep-th/9304154.

[24] S. Deser, "No local Maxwell duality invariance," Class.Quant.Grav. 28 (2011) 085009, 1012.5109 .

[25] C. Bunster and M. Henneaux, "Sp(2n,R) electric-magnetic duality as off-shell symmetry of interacting electromagnetic and scalar fields," PoS HRMS2010 (2010) 028, 1101.6064.

[26] A. Saa, "Local electromagnetic duality and gauge invariance," Class.Quant.Grav. 28 (2011) 127002, 1101.3927.

[27] K. G. Wilson, "Confinement of Quarks," Phys.Rev. D10 (1974) 2445-2459.

[28] A. Y. Kitaev, "Fault tolerant quantum computation by anyons," Annals Phys. 303 (2003) 2-30, quant-ph/9707021. 
[29] M. Levin and X.-G. Wen, "Quantum ether: photons and electrons from a rotor model," Phys.Rev. B73 (2006) 035122, hep-th/0507118.

[30] A. Kitaev, "Toward Topological Classification of Phases with Short-range Entanglement," unpublished (2011) http://online.kitp.ucsb.edu/online/topomat11/kitaev/.

[31] Y.-M. Lu and A. Vishwanath, "Theory and classification of interacting 'integer' topological phases in two dimensions: A Chern-Simons approach," Phys.Rev. B86 (2012) 125119, 1205.3156.

[32] J. Sau, B. Swingle, and T. Senthil, "unpublished,".

[33] A. Y. Kitaev, "Unpaired Majorana fermions in quantum wires," Physics-Uspekhi 44 (2001), no. 10S 131, cond-mat/0010440v2.

[34] R. Thorngren, "Framed Wilson Operators on the Boundaries of Novel SPT Phases," 1404.4385.

[35] A. Kitaev, "On the Classification of Short-Range Entangled States," unpublished (2013) http://scgp.stonybrook.edu/archives/7874.

[36] B. Swingle and J. McGreevy, "Renormalization group constructions of topological quantum liquids and beyond," ArXiv e-prints (July, 2014) 1407.8203.

[37] A. Vishwanath and T. Senthil, "Physics of Three-Dimensional Bosonic Topological Insulators: Surface-Deconfined Criticality and Quantized Magnetoelectric Effect," Phys. Rev. X 3 (Feb, 2013) 011016, http://link.aps.org/doi/10.1103/PhysRevX.3.011016.

[38] C. Xu and T. Senthil, "Wave functions of bosonic symmetry protected topological phases," Phys. Rev. B 87 (May, 2013) 174412, http://link.aps.org/doi/10.1103/PhysRevB.87.174412.

[39] A. S. Schwarz, "The Partition Function of Degenerate Quadratic Functional and Ray-Singer Invariants," Lett.Math.Phys. 2 (1978) 247-252.

[40] A. S. Schwarz, "The Partition Function of a Degenerate Functional," Commun.Math.Phys. 67 (1979) 1-16.

[41] E. Witten, "Quantum Field Theory and the Jones Polynomial," Commun.Math.Phys. 121 (1989) 351. 
[42] S. Elitzur, G. W. Moore, A. Schwimmer, and N. Seiberg, "Remarks on the Canonical Quantization of the Chern-Simons-Witten Theory," Nucl.Phys. B326 (1989) 108.

[43] G. T. Horowitz, "Exactly Soluble Diffeomorphism Invariant Theories," Commun.Math.Phys. 125 (1989) 417.

[44] M. Blau and G. Thompson, "Topological Gauge Theories of Antisymmetric Tensor Fields," Annals Phys. 205 (1991) 130-172.

[45] G. T. Horowitz and M. Srednicki, "A QUANTUM FIELD THEORETIC DESCRIPTION OF LINKING NUMBERS AND THEIR GENERALIZATION," Commun.Math.Phys. 130 (1990) 83.

[46] E. Witten, "AdS / CFT correspondence and topological field theory," JHEP 9812 (1998) 012, hep-th/9812012.

[47] G. W. Moore, "Anomalies, Gauss laws, and Page charges in M-theory," Comptes Rendus Physique 6 (2005) 251-259, hep-th/0409158.

[48] D. Belov and G. W. Moore, "Conformal blocks for AdS(5) singletons," hep-th/0412167.

[49] S. A. Hartnoll, "Anyonic strings and membranes in AdS space and dual Aharonov-Bohm effects," Phys.Rev.Lett. 98 (2007) 111601, hep-th/0612159.

[50] G. Moore, "Minicourse of three lectures on Generalized Abelian Gauge Theories, Self-Duality, and Differential Cohomology, at the Simons Center Workshop on Differential Cohomology, Simons Center for Geometry and Physics, Stonybrook," http://scgp.stonybrook.edu/archives/851.

[51] J. M. Maldacena, G. W. Moore, and N. Seiberg, "D-brane charges in five-brane backgrounds, Appendix A," JHEP 0110 (2001) 005, hep-th/0108152.

[52] D. S. Freed, G. W. Moore, and G. Segal, "Heisenberg Groups and Noncommutative Fluxes," Annals Phys. 322 (2007) 236-285, hep-th/0605200.

[53] X.-G. Wen, Quantum Field Theory of Many-Body Systems. Oxford Univ. Press, Oxford, 2004.

[54] R. Jackiw and C. Rebbi, "Spin from Isospin in a Gauge Theory," Phys.Rev.Lett. 36 (1976) 1116.

[55] R. Jackiw and C. Rebbi, "Solitons with Fermion Number 1/2," Phys. Rev. D13 (1976) 3398-3409. 
[56] E. Witten, "Dyons of Charge $\frac{e \theta}{2 \pi}$," Phys.Lett. B86 (1979) 283-287.

[57] J. Maciejko, X.-L. Qi, A. Karch, and S.-C. Zhang, "Fractional topological insulators in three dimensions," Phys.Rev.Lett. 105 (2010) 246809, 1004.3628.

[58] B. Swingle, M. Barkeshli, J. McGreevy, and T. Senthil, "Correlated Topological Insulators and the Fractional Magnetoelectric Effect," Phys.Rev. B83 (2011) 195139, 1005.1076.

[59] E. Witten, "On S duality in Abelian gauge theory," Selecta Math. 1 (1995) 383, hep-th/9505186.

[60] J. W. Milnor and J. D. Stasheff, Characteristic classes, volume 76 of Annals of Mathematics Studies. Princeton University Press, Princeton, NJ, 1974.

[61] O. Aharony, N. Seiberg, and Y. Tachikawa, "Reading between the lines of four-dimensional gauge theories," JHEP 1308 (2013) 115, 1305.0318.

[62] C. Vafa and E. Witten, "A Strong coupling test of S duality," Nucl.Phys. B431 (1994) 3-77, hep-th/9408074.

[63] T. Senthil and M. Levin, "Integer quantum Hall effect for bosons: A physical realization," Phys.Rev.Lett. 110 (2013) 046801, 1206.1604.

[64] A. Vishwanath and T. Senthil, "Physics of three dimensional bosonic topological insulators: Surface Deconfined Criticality and Quantized Magnetoelectric Effect," Phys. Rev. X 3, 011016 (2013) 1209.3058.

[65] J. T. Chalker and P. D. Coddington, "Percolation, quantum tunnelling and the integer Hall effect," Journal of Physics C Solid State Physics 21 (May, 1988) 2665-2679.

[66] P. Hosur, S. Ryu, and A. Vishwanath, "Chiral topological insulators, superconductors, and other competing orders in three dimensions," Phys. Rev. B 81 (Jan., 2010) 045120, 0908.2691.

[67] T. Neupert, C. Chamon, C. Mudry, and R. Thomale, "Wire deconstructionism and classification of topological phases," ArXiv e-prints (Mar., 2014) 1403.0953.

[68] M. A. Metlitski, C. L. Kane, and M. P. A. Fisher, "Bosonic topological insulator in three dimensions and the statistical Witten effect," Phys. Rev. B 88 (Jul, 2013) 035131, http://link.aps.org/doi/10.1103/PhysRevB.88.035131.

[69] N. Arkani-Hamed, A. G. Cohen, and H. Georgi, "(De)constructing dimensions," Phys.Rev.Lett. 86 (2001) 4757-4761, hep-th/0104005. 
[70] T. Banks, R. Myerson, and J. B. Kogut, "Phase Transitions in Abelian Lattice Gauge Theories," Nucl.Phys. B129 (1977) 493.

[71] G. 't Hooft, "On the Phase Transition Towards Permanent Quark Confinement," Nucl.Phys. B138 (1978) 1.

[72] G. 't Hooft, "A Property of Electric and Magnetic Flux in Nonabelian Gauge Theories," Nucl.Phys. B153 (1979) 141.

[73] G. 't Hooft, "Topology of the Gauge Condition and New Confinement Phases in Nonabelian Gauge Theories," Nucl.Phys. B190 (1981) 455.

[74] E. Witten, "An SU(2) anomaly," Phys. Lett. B117 (1982) 324-328.

[75] J. McGreevy and B. Swingle, "Non-Abelian statistics versus the Witten anomaly," Phys.Rev. D84 (2011) 065019, 1106.0004.

[76] J. Goldstone, "unpublished,".

[77] S. Elitzur and V. P. Nair, "NONPERTURBATIVE ANOMALIES IN HIGHER DIMENSIONS," Nucl. Phys. B243 (1984) 205.

[78] S. P. de Alwis, "ON THE RELATION BETWEEN GLOBAL AND U(1) ANOMALIES," Phys. Rev. D32 (1985) 2837.

[79] F. R. Klinkhamer, "Another look at the SU(2) anomaly," Phys. Lett. B256 (1991) 41-42.

[80] K.-H. Rehren, "BRAID GROUP STATISTICS AND THEIR SUPERSELECTION RULES," in The algebraic theory of superselection sectors, D. Kastler (ed.) Proceedings Palermo 1989 (1989).

[81] J. Frohlich and F. Gabbiani, "Braid statistics in local quantum theory," Rev.Math.Phys. 2 (1991) 251-354.

[82] A. Kitaev, "Anyons in an exactly solved model and beyond," Annals of Physics 321 (2006), no. 12 -111, cond-mat/0506438.

[83] M. A. Metlitski, L. Fidkowski, X. Chen, and A. Vishwanath, "Interaction effects on 3D topological superconductors: surface topological order from vortex condensation, the 16 fold way and fermionic Kramers doublets," ArXiv e-prints (June, 2014) 1406.3032.

[84] D. B. Kaplan, "Chiral Symmetry and Lattice Fermions," 0912.2560. 
[85] D. B. Kaplan and S. Sun, "Spacetime as a topological insulator: Mechanism for the origin of the fermion generations," Phys.Rev.Lett. 108 (2012) 181807, 1112.0302.

[86] X.-G. Wen, "A lattice non-perturbative definition of an $S O(10)$ chiral gauge theory and its induced standard model," Chin.Phys.Lett. 30 (2013) 111101, 1305.1045.

[87] Y.-Z. You, Y. BenTov, and C. Xu, "Interacting Topological Superconductors and possible Origin of $16 n$ Chiral Fermions in the Standard Model," ArXiv e-prints (Feb., 2014) 1402.4151.

[88] S. K. Donaldson and P. B. Kronheimer, The Geometry of Four-Manifolds. Oxford Univ. Press, Oxford, 1990.

[89] F. J. Wegner, "Duality in Generalized Ising Models and Phase Transitions without Local Order Parameters," Journal of Mathematical Physics 12 (1971), no. 10 2259-2272, http://scitation.aip.org/content/aip/journal/jmp/12/10/10.1063/1.1665530.

[90] T. Senthil and O. Motrunich, "Microscopic models for fractionalized phases in strongly correlated systems," Phys. Rev. B 66 (Nov, 2002) 205104.

[91] M. A. Levin and X.-G. Wen, "String-net condensation: A physical mechanism for topological phases," Phys. Rev. B 71 (Jan, 2005) 045110.

[92] C. W. von Keyserlingk, F. J. Burnell, and S. H. Simon, "Three-dimensional topological lattice models with surface anyons," Phys. Rev. B 87 (Jan., 2013) 045107, 1208.5128.

[93] T. H. Hansson, V. Oganesyan, and S. L. Sondhi, "Superconductors are topologically ordered," Annals of Physics 313 (Oct., 2004) 497-538, arXiv: cond-mat/0404327. 